K. TOHGE

KODAI MATH. J.

13 (1990), 409-416

\title{
ON THE VALUE DISTRIBUTION OF AN ENTIRE FUNCTION OF ORDER AT MOST ONE
}

\author{
By KazUYA TOHgE
}

\section{§1. Introduction.}

As a consequence of results on solutions to a differential equation $w^{\prime \prime}+A w$ $=0$, where $A$ is entire, Shen [5] and Rossi [4] proved a curious result:

There does not exist a transcendental entire function $E$ of order $\rho(E)<1$ such that the value of $E^{\prime}(z)$ at every zero of $E(z)$ is \pm 1 .

On proving this, they used the lemma of Bank and Laine [1] which states that such a function $E$ would have to be the product of two linearly independent solutions of the above second order differential equation. It follows from the counter-example given by Rossi, $E(z)=2 \sqrt{z} \sin \sqrt{z}$, that the conclusion can not hold even if only one zero fails to satisfy the assumption.

In this note we prove

THEOREM. Let $E(z)$ be a transcendental entire function of order $\rho(E) \leqq 1$ and $Q(z) \not \equiv$ a rational function. Suppose that $E^{\prime}(z)-Q(z)$ vanishes at every zero of $E(z)$ with possibly finitely many exceptions. Then $\rho(E)=1$ and further $E$ is of regular growth, and also the meromorphic function

$$
A(z)=\frac{E^{\prime}(z)-Q(z)}{E(z)}
$$

is one of the followings:

a) $A$ is a rational function such that for some nonzero constant $a, A(z) \rightarrow a$ as $z \rightarrow \infty$;

b) $A$ is a transcendental function of regular growth with $\rho(A)=1$, and has a finite number of poles.

This result may be read as a result on the zeros of $E^{\prime}(z)$.

We can easily give examples for the case a).

Example 1. For any polynomials $p \not \equiv 0$ and $q$ and also a nonzero constant

Received November 6, 1989; revised May 15, 1990. 
$a, E(z)=p(z)(\exp (a z)+q(z))$ and $Q(z)=p(z)\left(q^{\prime}(z)-a q(z)\right)$ satisfy the hypotheses of our theorem and then

$$
A(z)=\frac{p^{\prime}(z)+a p(z)}{p(z)} \longrightarrow a, \quad \text { as } z \rightarrow \infty .
$$

Example 2. For the entire function $E(z)=\left(e^{z}-1\right) / z$ and the rational function $Q(z)=1 / z$ we have $A(z)=(z-1) / z$ given by $(1.1)$.

Also for the case b) we have

Example 3. The entire function $E(z)=2 \sqrt{z} \sin \sqrt{z} \cdot \exp (i z / \pi)$ and $Q(z) \equiv 1$ satisfy the hypotheses of our theorem. Then we have

$$
A(z)=-\frac{1}{E(z)}+\frac{(\sin \sqrt{z})^{\prime}}{\sin \sqrt{z}}+\frac{1}{2 z}+\frac{i}{\pi},
$$

and (see $[2:$ p. 7$]$ )

$$
m(|z|, A) \sim m(|z|, 1 / E) \sim m\left(|z|, e^{\imath z / \pi}\right)=|z| / \pi^{2}, \quad \text { as }|z| \rightarrow \infty .
$$

Example 4. The entire function $E(z)=e^{-2 z}\left(z-e^{z}\right)$ and the rational function $Q(z)=(1-z) / z^{2}$ imply the meromorphic function

$$
A(z)=\left\{(1-z) e^{z}+z(1-2 z)\right\} / z^{2}
$$

from the definition (1.1).

Of course, the theorem does not hold in general for meromorphic $E(z)$, since the function $A(z)$ as in (1.1) has always the poles possibly except for those of $Q(z)$ wherever the function $E(z)$ does. If $E(z)$ has, however, only a finite number of poles, the corresponding result to this theorem is easily obtained.

\section{§2. Preliminaries.}

To prove the theorem we make a direct application of a method of Rossi in [4]. It bases on the Beurling-Tsuji estimate for harmonic measure and needs the following three lemmas proved there.

LEMMA 1. Let $E$ be an entire function of finite order. Given $\varepsilon>0$ there exists a constant $C=C(\varepsilon)$ such that

$$
\left|\frac{E^{\prime}}{E}\left(r e^{i \theta}\right)\right| \leqq r^{C},
$$

for all $r \geqq r_{0} \geqq 1$ and all $\theta \notin J(r)$, where the angular measure of the set $J(r)$ in $[0,2 \pi), m(J(r))$ is $\leqq \varepsilon \pi$.

To state the second lemma we need some notation. Let $D$ be a region in the 
complex plane $C$. We denote by $\theta_{D}(r)$ the measure of all $\theta$ in $[0,2 \pi)$ such that $r e^{i \theta} \equiv D$. To each $r \geqq 1$, if the entire circle $|z|=r$ lies in $D$, set $\theta_{D}^{*}(r)=+\infty$; otherwise $\theta_{D}^{*}(r)=\theta_{D}(r)$. As usual, the order $\rho(u)$ and the lower order $\mu(u)$ of a function $u(z)$ subharmonic in $\boldsymbol{C}$ are given by

$$
\rho(u)=\varlimsup_{r \rightarrow \infty} \frac{\log M(r, u)}{\log r} \text { and } \mu(u)=\varliminf_{r \rightarrow \infty} \frac{\log M(r, u)}{\log r},
$$

where $M(r, u)=\sup _{|z|=r} u(z)$. Also for an entire function $E(z)$, they are given by $\rho(E)=\rho(\log |E|)$ and $\mu(E)=\mu(\log |E|)$. Then we have

Lemma 2. Let $u$ be a subharmonic function in $C$ and let $D$ be an open component of $\{z: u(z)>0\}$. Then

$$
\begin{aligned}
& \rho(u) \\
& \mu(u)
\end{aligned} \varlimsup_{R \rightarrow \infty}(\log R)^{-1} \pi \int_{1}^{R} \frac{d t}{t \theta_{D}^{*}(t)} .
$$

Furthermore, given $\varepsilon>0$, define $F=\{r: \theta *(r) \leqq \varepsilon \pi\}$. Then

$$
\varlimsup_{R \rightarrow \infty}(\log R)^{-1} \int_{F \cap[1, R]} d t / t \leqq \varepsilon \rho(u) .
$$

In this lemma we shall make a minor modification to $\theta_{D}^{*}(r)$ when $\theta_{D}(t)=0$, $1 \leqq t \leqq t_{0}$.

LEMMA 3. Let $l_{1}(t)$ and $l_{2}(t)$ be two positive and measurable functions on $[1, \infty)$ with $l_{1}(t)+l_{2}(t) \leqq(2+\varepsilon) \pi$, where $\varepsilon>0$. If $G \subset[1, \infty)$ is any measurable set and

$$
\pi \int_{t l_{2}(t)}^{d t} \leqq \alpha \int_{G} d t / t, \quad \alpha \geqq 1 / 2
$$

then

$$
\pi \int \frac{d t}{t l_{1}(t)} \geqq \frac{\alpha}{(2+\varepsilon) \alpha-1} \int_{G} d t / t
$$

\section{§ 3. Proof of Theorem.}

Now the function $A(z)$ given by (1.1) is regular at every simple zero of $E(z)$ possibly with finitely many exceptions. The poles of $A(z)$ may therefore occur only at multiple zeros of $E(z)$ or poles of $Q(z)$. The number of these points is however at most finite since our assumption requires the rational function $Q(z)$ should vanish at each multiple zero of $E(z)$ except for finitely many. Thus $A(z)$ is a meromorphic function having only a finite number of poles.

We now distinguish the cases whether $A$ is rational or transcendental.

$C A S E 1$; in which $A$ is rational. The entire function $E(z)$ considered here is a solution to the non-homogeneous differential equation 


$$
w^{\prime}=A(z) w+Q(z)
$$

with the rational coefficients, We apply the Wiman-Valiron theory (see $[6: \mathrm{p}$. 105 , Theorem 30]) to the equation with $w=E(z)$ and note that $E(z)$ is now transcendental. Then the central index $n(r)$ satisfies the relation

$$
n(r)=r\left|A\left(z_{r}\right)\right|\{1+o(1)\}
$$

as $r$ tends to infinity outside a set $\Delta$ of finite logarithmic measure, $m_{l}(\Delta)$, where $z_{r}$ is a point at which $\left|E\left(z_{r}\right)\right|=\max _{|z|=r}|E(z)|$ and $\left|z_{r}\right|=r$. The rational function $A(z)$ has the asymptotic representation

$$
A(z)=a z^{m}\{1+O(1 /|z|)\}, \quad(z \rightarrow \infty)
$$

for some nonzero constant $a$ and an integer $m$. Thus (3.2) gives

$$
\rho(E) \geqq \varlimsup_{\substack{r \rightarrow \infty \\ r \notin \notin \Delta}} \frac{\log n(r)}{\log r}=m+1 .
$$

Since the right-hand side is non-negative and $\rho(E) \leqq 1$, we have $m=0$ or $m=-1$. The former implies $\rho(E)=1$, and further we can see $\mu(E)=1$. In fact, if $r \in \Delta$ and $\log r>m_{l}(\Delta)+1$, then there exists a $\tau=\tau(r)$ with $\exp \left(-m_{l}(\Delta)-1\right) \leqq \tau<1$ such that $\tau r \notin \Delta$. Thus it follows from monotonicity of $n(r)$ (see [6]) that $n(r) \geqq$ $n(\tau r) \geqq n\left(\exp \left(-m_{l}(\Delta)-1\right) r\right)$. Hence we have

$$
\log n(r)=(1+o(1)) \log r, \quad \text { as } r \rightarrow \infty .
$$

It is easy to see that $m(r, 1 / E) \leqq m(r, 1 / Q)+m\left(r, E^{\prime} / E\right)+m(r, A)+\log 2=O(\log r)$. These results give the case a) as in our theorem. Next we suppose $m=-1$. Then we transform (3.1) into the equation

$$
y^{\prime}+\frac{1}{\zeta^{2}} A(1 / \zeta) y+\frac{1}{\zeta^{2}} Q(1 / \zeta)=0,
$$

by setting $y(\zeta)=w(1 / \zeta)$. Fix $r>0$ sufficiently small and let $D$ be the simply connected domain $\{\zeta: 0<|\zeta|<r, 0<\arg \zeta<2 \pi\}$. Then a solution to (3.4) in $D$ is given by

$$
y\left(\zeta ; \zeta_{0}, y_{0}\right)=\exp \left(-\int_{\zeta_{0}}^{\zeta} \frac{1}{s^{2}} A(1 / s) d s\right)\left(y_{0}-\int_{\zeta_{0}}^{\zeta} \frac{1}{t^{2}} Q(1 / t) \exp \left\{\int_{\zeta_{0}}^{t} \frac{1}{s^{2}} A(1 / s) d s\right\} d t\right)
$$

where $\zeta_{0} \in D$ and $y_{0} \Subset \boldsymbol{C}$ (see [3]). In the domain $D$, we may write

$$
\begin{aligned}
& \zeta^{-2} A\left(\zeta^{-1}\right)=a \zeta^{-1}\{1+O(|\zeta|)\}, \\
& \zeta^{-2} Q\left(\zeta^{-1}\right)=b \zeta^{-(k+2)}\{1+O(|\zeta|)\}, \quad b \in C-\{0\}, k \text { integer, }
\end{aligned}
$$

and thus for a constant $d_{0}$

$$
y\left(\zeta ; \zeta_{0}, y_{0}\right)=e^{-d_{0} \zeta^{-a}}\{1+O(|\zeta|)\}\left(y_{0}-e^{d} 0 b\{1+O(|\zeta|)\} \int_{\zeta_{0}}^{\zeta} t^{-(k-a+2)} d t\right),
$$


as $|\zeta| \rightarrow 0$ there. The function $E(1 / \zeta)$ posesses the above representation and by its monodromy property about the origin then it follows that either the constant $a$ must be an integer or the coefficient of $\zeta^{-a}$ in (3.5) must vanish identically in a neighborhood of the origin. Then $E(1 / \zeta)$ has the origin as possibly a pole, which implies that the entire function $E(z)$ cannot be transcendental. This is a contradiction and the completes the proof in this case.

CASE 2; in which $A$ is transcendental. From the reason given at the beginning of this section, we may now write $A(z)=B(z) / P(z)$ with an entire function $B(z)$ and a polynomial $P(z)$. Then we have

$$
B(z)=P(z)\left(\frac{E^{\prime}(z)}{E(z)}-\frac{Q(z)}{E(z)}\right)
$$

Let $k$ and $l$ be the degrees of the rational function $Q(z)$ and the polynomial $P(z)$, respectively. After the manner of Rossi, fix $\varepsilon>0$ and choose an integer $N$ such that

$$
N>\max (C, k)+l,
$$

where $C$ is the constant as in Lemma 1 and

$$
\log M(2, B)<N \log 2 .
$$

Since $B$ is transcendental there exists a point $z_{0},\left|z_{0}\right|>2$, such that $\log \left|B\left(z_{0}\right)\right|$ $>N \cdot \log \left|z_{0}\right|$. Let $D_{1}$ be the connected component of the set

$$
\{z: \log |B(z)|-N \cdot \log |z|>0\},
$$

containing $z_{0}$. By the choice of $N, \log |B(z)|-N \cdot \log |z|$ is harmonic in $D_{1}$ and identically zero on the boundary. Thus the function $u$ defined by

$$
u(z)=\left\{\begin{array}{cl}
\log |B(z)|-N \log |z| & \left(z \in D_{1}\right), \\
0 & \left(z \in C-D_{1}\right),
\end{array}\right.
$$

is subharmonic in $\boldsymbol{C}$ with the lower order and the order

$$
\mu(u) \leqq \mu(B) \quad \text { and } \quad \rho(u) \leqq \rho(B) .
$$

It is easily shown that $\rho(B)=\rho(A) \leqq \rho(E)$, which will be mentioned later.

Let $D_{2}$ be any connected component of $\{z: \log |E(z)|>0\}$ and let $D_{3}=$ $\left\{r e^{i \theta}: \theta \in J(r)\right\}$ where $J(r)$ is as in Lemma 1. If the set $\left(D_{1} \cap D_{2}\right)-D_{3}$ contains an unbounded sequence $r_{n} \ell^{i \theta_{n}}, n=1,2, \cdots$, we obtain from the definitions of $D_{1}, D_{2}$, and $D_{3}$, Lemma 1 and also (3.6)

$$
r_{n}{ }^{N} \leqq\left|B\left(r_{n} e^{i \theta_{n}}\right)\right| \leqq\left|P\left(r_{n} e^{i \theta_{n}}\right)\right|\left\{r_{n}{ }^{C}+\left|Q\left(r_{n} e^{i \theta_{n}}\right)\right|\right\}, \quad r_{n} \geqq r_{0} .
$$

This clearly contradicts (3.7) for $n$ large enough. 
Then for arbitrary fixed $\varepsilon>0$, we may assume that $\left(D_{1} \cap D_{2}\right)-D_{3}$ is bounded. This implies that for $r \geqq r_{1} \geqq r_{0}$

$$
K(r)=\left\{\theta: r e^{i \theta} \in D_{1} \cap D_{2}\right\} \subset J(r),
$$

and thus by Lemma 1 the angular measure of $K(r)$ satisfies

$$
m(K(r)) \leqq \varepsilon \pi .
$$

Setting $l_{j}(r)=\theta_{D_{j}}(r)$ given in $\S 2$, we have $l_{j}(r)>0$ for $r$ sufficiently large since each $D_{j}$ is an unbounded domain, $j=1,2$. Also (3.9) gives $l_{1}(r)+l_{2}(r) \leqq$ $(2+\varepsilon) \pi\left(r \geqq r_{1}\right)$. If need were, by putting $\theta_{D_{j}}(r) \equiv \pi(j=1,2)$ for $r<r_{1}$, we could assume each $l_{j}(r)>0(j=1,2)$ and this inequality to be true for any $r \geqq 1$. Now let us set

$$
\varlimsup_{R \rightarrow \infty}(\log R)^{-1} \pi \int_{1}^{R} \frac{d t}{t l_{2}(t)}=\alpha .
$$

By the definition of the $l_{2}, \alpha \geqq 1 / 2$. Since $l_{1}$ and $l_{2}$ satisfy the hypotheses of Lemma 3, we obtain

$$
\varliminf_{R \rightarrow \infty}(\log R)^{-1} \pi \int_{1}^{R} \frac{d t}{t l_{1}(t)} \geqq \frac{\alpha}{(2+\varepsilon) \alpha-1} .
$$

Define $B_{\jmath}=\left\{r: \theta_{D_{j}}(r)=\infty\right\}$ and $\tilde{B}_{j}=[1, \infty)-B_{\jmath}, j=1,2$. If $r \in B_{1}$, we have $\theta_{D_{2}}^{*}(r) \leqq \varepsilon \pi$ by (3.9). Thus $B_{1} \subset\left\{r: \theta_{D_{2}}^{*}(r) \leqq \varepsilon \pi\right\}$ and similarly $B_{2} \subset\left\{r: \theta_{D_{1}}^{*}(r) \leqq \varepsilon \pi\right\}$. By (2.2) we have

$$
\varlimsup_{R \rightarrow \infty}(\log R)^{-1} \int_{B_{2 \cap[1, R]}} d t / t \leqq \varepsilon \rho(u) .
$$

Then (2.1), (3.10) and (3.12) give

$$
\begin{aligned}
& \rho(E) \geqq \varlimsup_{R \rightarrow \infty}(\log R)^{-1} \pi \int_{1}^{R} \frac{d t}{t \theta \mathcal{D}_{2}(t)} \\
&=\varlimsup_{R \rightarrow \infty}(\log R)^{-1} \pi \int_{\tilde{B}_{2 \cap[1, R]}} \frac{d t}{t l_{2}(t)} \\
&=\varlimsup_{R \rightarrow \infty}(\log R)^{-1}\left(\pi \int_{1}^{R} \frac{d t}{t l_{2}(t)}-\frac{1}{2} \int_{B_{2 \cap[1, R]}} d t / t\right) \\
& \geqq \alpha-(\varepsilon / 2) \rho(u) .
\end{aligned}
$$

While by (2.2) we have

$$
\varlimsup_{R \rightarrow \infty}(\log R)^{-1} \int_{B_{1 \cap[1, R]}} d t / t \leqq \varepsilon \rho(E),
$$

and by (3.8), (2.1), (3.11) and (3.14) we obtain 


$$
\begin{aligned}
\mu(A) & =\mu(B) \geqq \mu(u) \geqq \lim _{R \rightarrow \infty}(\log R)^{-1} \pi \int_{1}^{R} \frac{d t}{t \theta \mathcal{D}_{1}(t)} \\
& =\lim _{R \rightarrow \infty}(\log R)^{-1}\left(\pi \int_{1}^{R} \frac{d t}{t l_{1}(t)}-\frac{1}{2} \int_{B_{1 \cap[1, R]}} d t / t\right) \\
& \geqq \frac{\alpha}{(2+\varepsilon) \alpha-1}-(\varepsilon / 2) \rho(E) .
\end{aligned}
$$

Inequalities (3.13) and (3.15) give then

$$
\mu(A) \geqq \frac{\rho(E)+(\varepsilon / 2) \rho(u)}{(2+\varepsilon)\{\rho(E)+(\varepsilon / 2) \rho(u)\}-1}-(\varepsilon / 2) \rho(E) .
$$

Since $\varepsilon$ was arbitrarily chosen and $\rho(u) \leqq \rho(E)$ we obtain the inequality

$$
\mu(A) \geqq \frac{\rho(E)}{2 \rho(E)-1}
$$

and thus

$$
\mu(A)^{-1}+\rho(E)^{-1} \leqq 2 .
$$

On the other hand, (3.6) implies easily

$$
m(r, A)=m(r, 1 / E)+O(\log r) \quad(r \rightarrow \infty)
$$

and therefore

$$
T(r, A)+N(r, 1 / E)=T(r, E)+O(\log r) \quad(r \rightarrow \infty) .
$$

This shows $\rho(A) \leqq \rho(E)$ as previously mentioned. Since $\rho(E) \leqq 1$, (3.16) implies $\mu(A) \geqq 1$ and thus $\mu(A)=\rho(A)=\rho(E)=1$. By interchanging the roles of $E$ and ( $u$ rather than) $A$ the above discussion yields

$$
\mu(E)^{-1}+\rho(A)^{-1} \leqq 2,
$$

and thus $\mu(E)=1$. Hence we obtain the case b) and the proof of Theorem is completed.

\section{REFERENCES}

[1] BANK, S.B. AND I. LAINE, On the zeros of meromorphic solutions of secondorder linear differential equations, Comment. Math. Helv. 58 (1983), 656-677.

[2] Hayman, W.K., Meromorphic functions, Clarendon Press, Oxford, 1964.

[3] HiLle, E., Ordinary differential equations in the complex domain, Wiley, New York, 1976.

[4] Rossi, J., Second order differential equations with transcendental coefficients, Proc. Amer. Math. Soc. 97 (1986), No. 1, 61-66.

[5] SHEN, L.C., Solution to a problem of S. Bank regarding exponent of convergence 
of zeros of the solutions of differential equation $f^{\prime \prime}+A f=0$, Keuxue Tongbao 30 (1985), No. 12, 1579-1585.

[6] VALIRON, G., Lectures on the general theory of integral functions, translated by E. F. Collingwood, Chelsea, New York, 1949.

Department of Mathematics

ScIENCE UNIVERsity OF TOKYO

Noda, Chiba, JapaN 